\title{
Review on Learning Parity with Noise based cloud computing
}

\author{
Tarasvi Lakum ${ }^{1}$, B.Thirumala Rao $^{2 *}$ \\ ${ }^{1}$ Research Scholar, Department of CSE, Koneru Lakshmaiah Education Foundation, Vaddeswaram, A.P, India, \\ ${ }^{1}$ tarasiru1@gmail.com \\ ${ }^{2 *}$ Professor, Department of CSE, Lakireddy Bali Reddy College of Engineering, Mylavaram, India \\ 2*drbtrao@lbrce.ac.in
}

\begin{abstract}
One of the emerging researches is cloud computing, in this area crypto-graphical scheme, generating public key, using "Learning Parity with Noise" or some advances in "LPN" schemes required. The existing systems still have problems due to decryption failures that have to be fixed. Herein we are reviewing the limitations and advantages of the various LPN Techniques.
\end{abstract}

Key words : Cloud computing, public-key, Parity, encryption

\section{INTRODUCTION}

In the case of spontaneous classification noise, a marginally sub exponential time algorithm for learning parity functions, a topic closely related to several cryptographic and coding issues. In the case of parity functions that depend only on the first $\mathrm{O}(\log \mathrm{n} \log \mathrm{n})$ bits of data, our algorithm runs in polynomial time, which offers the first known instance of an effective noise tolerant algorithm for a principle class that cannot be studied in the Kearns Statistical Query model. The set of problems that can be discovered in the mathematical query model is a specific subset of those problems that can be studied in the PAC model's presence of noise. In coding-theory terms is a poly(n)-time algorithm for decoding linear $\mathrm{k} £ \mathrm{n}$ codes in the presence of random noise in the case of $k \mathrm{D} c \log n \log n$ for some $\mathrm{c}>0$. (The case of k D O $(\log n$ ) is trivial since one can check each of the possible $2 \mathrm{k}$ messages individually and choose the one that gives the nearest codeword). A natural extension of the statistical query model is to allow queries of statistical properties involving t-tuples of examples, as opposed to single examples only. The second result of this article is to prove that any class of functions that can be trained (strongly or weakly) with $t$-wise queries for $\mathrm{t} D$ $\mathrm{O}(\log \mathrm{n})$ with regular unary queries is also weakly learnable. Therefore, this natural extension to the mathematical query model does not expand the collection of functions that are weakly trained [1].

The LPN-C is a probabilistic private key encryption method, the security of which can be reduced to an LPN issue for learning difficulty. The suggested protocol only includes basic GF(2) operations and the form of error correction. This indicates that this is invisible in adaptive plaintext attacks (IND-P2-C0). To hold the computer free in adaptive attacks chosen by ciphertext, the secure MAC is attached. This software extends the collection of available cryptographic primitives that rely on the difficulty of the LPN [2].

A well-known hard problem researched in cryptography and philosophy of coding is Understanding Parity with Noise Problems(LPN). The LPN problem is thought to be resistant to quantum computers and to be an alternative to other numerical issues (such as factorization and discreet logarithms) which can easily be solved on quantum computers. It is also a good candidate for lightweight appliances, because of its simplicity.

The LPN problem in this writing along with its cryptography implementations is also, rely on LPN algorithms to overcome them. Aim is to see what are the lower limitations to solve the problem, and to create a fully homomorphic LPN-based encryption scheme by using appropriate parameters. LPN is believed to be quantum computers immune. The right parameters for the LPN questions are, we intend to expand current LPN cryptosystems. LWE-based encryption schemes [8] and several lattice-based schemes already exist in fully homomorphic form. A fully homomorphic scheme makes any cipher-text procedure without the need of the awareness of the secret key such that the decrypted output is the effect on the corresponding plaintexts of the procedure itself. To see what techniques are required to convert a completely homomorphic LPN encryption method [3].

When studying noise problem parity well learned in theory and cryptography of research, we have access to an oracle which, when pressing a button, returns a $E \mathrm{GF}(2)^{\mathrm{n}}$ random vector with a bit $b \boldsymbol{e} \mathrm{GF}$ (2) computed as a.u+ $\eta$, where $u \boldsymbol{\epsilon}$ $\mathrm{GF}(2)^{n}$ are a hidden vector, and $\eta \in \mathrm{GF}$ (2) is a noise bit which is 1 with a certain chance of $p$. Say it $p=1 / 3$. The aim is to restore you. This mission is unlikely to be uncompromising. Here we present a slight(?) variant: we obtain 10 random vectors a1, a2, ..., a10 $\epsilon \mathrm{GF}(2)^{n}$, and corresponding bits $b 1, b 2, \ldots ., b 10$, of which at most 3 are noisy. The oracle will determine which of the 10 bits is noise. We display a polynomial time algorithm for the recovery of the hidden vector $\mathrm{u}$.

We talk about basic effects and also about studying of more common noise patterns. In this standardized noise model, we 
can also learn low-depth decision trees. Also, in our organized noise settings we consider learning with error problem over $\mathrm{GF}(\mathrm{q})$ and (a) giving a $2^{\delta \sqrt{m}}$ algorithm a slightly under-exponent algorithm (b)(4).

They take into consideration the issue of studying diffuse noise balance. An algorithm which runs on time poly $\log \left(\frac{1}{n}, \frac{1}{1-2 n}\right) n^{\left(1+(2 n)^{2}+0(1)\right.} \frac{r}{2}$ and uses only $\frac{\operatorname{rog}\left(\frac{n}{n}\right)[1]}{(1-2 n)^{2}}$ for learning parity in $r$ out of $n$ variables.

This method operates with harmful noise from previously established experiments and generalizes it to random distributions.

Although efficient algorithms in the presence of noise will have a significant impact in learning certain groups of theories, our research is the first to provide a connection that is greater than the brute force $\mathrm{O}\left(n^{r}\right)$.

Because of this, we achieve the first non-trivial relation for the existence of noise to learn r-Juntas, and a slight increase in the sophistication of the standardized distribution of DNF instruction. In order to know together there are immediate concerns as to whether the non-trivial relationship can be expanded from Corollary 2 to arbitrary distribution and, in turn, whether the running time for $n^{E T}$ can be raised, to a constant $c<1$. As before, a significant question open is that there is a polynomial learning time algorithm $\omega(1)$ - juntas [5].

A variety of crypto-graphical implementations were identified recently in the Learning Parity with Noise (LPN) issue as the hardness principle of "possibly secure" crypto-graphical schemes such as encryption or authentication protocols. The established reliability of the device ensures the proof demonstrates that the presence of an effective adversary to the scheme suggests a misunderstanding of the underlying hardness. Theoretical and functional explanations are important to LPN-based schemes. LPN-based devices give a very good protection assurance on the theoretical side.

The LPN is equal to the problem that has been researched widely over the fifty years, the encoding to random linear codes. The fastest known algorithms operate indefinitely, and the LPN problem does not lead to existing quantum algorithms, as compared to other numerological cryptographic problem. At the functional hand, code-size and space-based LPN systems are always incredibly easy and efficiently. It renders them leading candidates for lightweight devices such as RFID tags, too poor for the application of common cryptographic primitives, such as the AES block counter.

A seamless transition to proven protection by easy LPN-based systems. The new public identity houses, agreements and zero-knowledge shows are focused on the pseudorandom generators and the symmetrical key cryptography, secure authentication protocols and if time permits. A common theory in the field of hidden encryption is that it is not feasible for safe networks to contend with unique buildings like the AES Block Cyclist. This perspective is at least questioned by recent constructions based on the hardness of LPN. An effective LPN block recycler (and block recyclers are used for virtually any main task), build for main tasks like verification, detection and authentication for messages.

The established protection is not only a pleasant theoretical benefit in certain settings (mostly for lightweight devices like RFIDs) but can potentially contribute to buildings that surpass the utility versus functional protection recognized dedicated systems (a point of view that is widely agreed in the field of pubic key cryptography [6].

The resultant crypto system can be indistinguishable in selected plaintext attacks (IND-CPA security). HELEN, a modern public-key code-based crypto-system whose protection is focused on the hardness of the Parity with Noise Issue (LPN) learning and the definitive minimum distance question. HELEN achieves IND-CCA protection in the random oracle model with the default Fujisaki-Okamoto architecture [7].

This article discusses the difficulties of computing sparse solutions in F2 linear equations structures. Consider the question k-EVENSET: given a standard linear equations scheme of $\mathrm{F} 2$ in $\mathrm{n}$ variables, determine if Hamming weight solution occurs at most $\mathrm{k}$ (i.e. k-sparse solution). A nonzero solution exists, while it has a straightforward $\mathrm{O}\left(\mathrm{n}^{\mathrm{k} / 2}\right)$ time algorithm, it is infamous that k-EVENSET has a fixed parameter intractability. K-EVENSET has no poly(n) $2^{o(\sqrt{h)}}$ (always) time algorithm for all $\mathrm{k}$ unless it can solve $\mathrm{k}$-clique in $\mathrm{n}^{\mathrm{o}(\mathrm{k})}$ time and no polynomial time algorithm unless $\mathrm{k}$-calculation is necessary $\mathrm{k}=\omega\left(\log ^{2} \mathrm{n}\right)$ [8].

An analysis of public-key cryptosystems focused on the LPN (Learning Parity with Noise) question variations. Alekhnovich developed the first LPN form in consideration (FOCS 2003), and explain some changes to the originally proposed structure, influenced by specific current versions of the LWE-based cryptosystem in Regev. The first public-key cryptosystem based on the ring-LPN problem, which is a more recently introduced LPN version, which results in substantial improvements in both time and space, in order to achieve further elevation. A variant of this issue called the Ring-LPN transposed problem. Far more successful is the public-key scheme focused on this problem. In practice, despite the best commonly established attacks, the requirements necessary for the specific protection levels.

The simple LPN-based system is not compatible with current realistic systems in several ways, as the public key, ciphertexts and encryption period for 80-bit authentication is already becoming very high. On the other side, in all of these ways, the scheme based at transposed Ring- LPN is far stronger. Although the public key and ciphertexts are still greater at comparable security levels than for, say, RSA, they are not prohibitively big; In comparison, the decryption scheme outperforms RSA for protection rates of 112 bits or greater. Nevertheless, the Ring-LPN-based system is less elegant. Therefore, public-key cryptography based on LPN seems to be somewhat more feasible for practical use than was generally assumed up to now. 
Tarasvi Lakum et al., International Journal of Emerging Trends in Engineering Research, 8(10), October 2020, 6859 - 6863

\begin{tabular}{|c|c|c|c|c|c|c|}
\hline Security level (bits) & $\begin{array}{l}\text { Time per } \\
80\end{array}$ & $\begin{array}{l}\text { Encryption } \\
112\end{array}$ & $\begin{array}{l}(\mathrm{ms}) \\
128\end{array}$ & $\begin{array}{l}\text { Time per } \\
80\end{array}$ & $\begin{array}{l}\text { decryption } \\
112\end{array}$ & $\begin{array}{l}(\mathrm{ms}) \\
128\end{array}$ \\
\hline $\begin{array}{l}\text { Basic LPN cryptosystem } \\
\text { Basic TRLPN cryptosystem }\end{array}$ & $\begin{array}{l}25.400 \\
1.100\end{array}$ & $\begin{array}{l}127.600 \\
2.250\end{array}$ & $\begin{array}{l}239.900 \\
3.200\end{array}$ & $\begin{array}{l}0.004 \\
\text { “ }\end{array}$ & $\begin{array}{l}0.007 \\
\text { “ }\end{array}$ & $\begin{array}{l}0.008 \\
\text { “ }\end{array}$ \\
\hline $\begin{array}{l}\text { Multi-bit LPN } \\
\text { Multi-bit TRLPN } \\
\text { Ring-LPN cryptosystem }\end{array}$ & $\begin{array}{l}25.800 \\
1.400 \\
13.200\end{array}$ & $\begin{array}{l}128.400 \\
3.100 \\
29.900\end{array}$ & $\begin{array}{l}241.700 \\
4.400 \\
42.200\end{array}$ & $\begin{array}{l}0.052 \\
، \\
3.100\end{array}$ & $\begin{array}{l}0.098 \\
\text { “ } \\
6.900\end{array}$ & $\begin{array}{l}0.128 \\
“ \\
9.700\end{array}$ \\
\hline RSA & 0.010 & 0.030 & 0.060 & 0.140 & 0.940 & 2.890 \\
\hline
\end{tabular}

\begin{tabular}{|l|l|l|l|l|}
\hline Security level (bits) & $\mathrm{n}$ & $\mathrm{T}$ & $\mathrm{A}$ & $\mathrm{b}$ \\
\hline 80 & 150000 & 0.00024 & 18 & 13 \\
112 & 350000 & 0.00016 & 38 & 13 \\
128 & 500000 & 0.00013 & 59 & 17 \\
196 & 1500000 & 0.000099 & 65 & 18 \\
256 & 2700000 & 0.000057 & 42 & 20 \\
\hline
\end{tabular}

\section{Encryption/decryption times for comparison}

Parameters for selected security parameters for ring-LPN cryptosystem [9]. The security of the scheme is based on the known clique and noise parity. The relevance of this method is justified by its Post-Quantum nature. No known quantum attacks are against our Candidate system, unlike the RSA and other cryptosystems based on the factorizing hardness or on the discrete logarithm that can be broken through a suitable large quantum device. A public key encoding scheme whose reliability is based on the clique's difficulty and is secure against quantum algorithms. However, a variety of enhancements is expected. Our key size at present is $n 2 / 2$. Thus, we expect to boost the algorithm in further progress, as it is now the same as the graph scale, which really is a great overhead for the encrypted document. The huge size of the key will be insignificant in operation as it just has to be moved once. Work in Jerrum [Jer92] will analyze the $2 \log (\mathrm{n})<\mathrm{k}$ $<\sqrt{ } n$ interval for hidden scaling as the finding toughness of an independent set increases exponentially with the independent set, as can be shown in previous parts. Look forward to further formal proof of safety in the present work and further experiments in the future [10].

A firmest health evaluation of LPN-based fault attack implementations, the key conclusion is that these systems have intrinsically strong features to withstand such assaults. Second, other popular fault models are useless against LPN (e.g. when an attacker turns bits in an implementation). Furthermore, attacks utilizing more complex fault models (e.g. where the attacker sets bits in an implementation) need much more tests than regular symmetrical rudimentary cryptographs such as block ciphers. Inaccurate insertions of faults trigger a severe identification of the severity of such assaults.
Along with previous observation of the fascinating algebraic structure for side channel resistance through the masking of internal products computed in LPN implementations, these findings therefore imply that primitive LPNs are important candidates for physically safe implementation [11].

The common equivalent of NP Complete "complete linear codes" is Learning Parity with Noise (LPN) and has been researched thoroughly in learning theory and encoding with applications to cryptography that react to the use of quantum systems. Sparse LPN version with a sparse matrix (or with of dimension of the matrix following the Bernoulli distribution), the version that Benny, Boaz and Avi have in mind (STOC 2010) comes under a specific (extreme) category.

For win-win that at least one of these is right: (1) the toughness of sparse LPN is implied in that of regular LPN with the same amount of noise;

(2) There is modern public encryption black-box constructions (PKE) and accidental transmission (OT) of regular LPN protocols. Non-trivial proof that the sparse LPN will be difficult as long as the regular LPN is difficult at the same level (or) otherwise it would contribute to more shocking (and possibly a breakthrought for other parameters) findings that public key encryptions and oblivious transmission protocols may be focussed on regular LPN at any noise rate and without any sub-exponential theorem of hardness [12].

In the modern quantum physics, it is of considerable importance to show the quantum advantage with less efficient yet more practical instruments. Recently, the question of studying a concealed parity function with noise was solved at considerable quantum level. Nonetheless, the algorithm would fail if all the data qubits were depolarized on the performance of the query. In this review, an algorithm for 
quantum parity learning that shows quantum gain when non-zero polarization is given to a qubit in each problem.

Under this case, quantum parity analysis obviously is qubit deterministic quantum computation. The concealed parity function will then be exposed by a series of operations, which can be interpreted as measurements of non-local observables of non-zero polarization and increasing data qubit. The origins from the resource-theoretical viewpoint of the quantum advantage in our algorithm. Although efforts are required to construct standard quantum computers that follow

\section{Comparison with Damgård Scheme and RSA public key encryption scheme.}

The design of security and practical public key encryption schemes is critical for protecting cyber security and privacy. Big data and cloud computing today not only bring unprecedented opportunities but also basic security challenges.

\begin{tabular}{|l|l|l|l|l|l|l|}
\hline & Time per & encryption & $(\mathrm{ms})$ & Time & per & Decryption \\
\hline Security level (bits) & 80 & 112 & 128 & 80 & 112 & 128 \\
\hline RSA scheme(not padding) & 0.010 & 0.030 & 0.060 & 0.140 & 0.940 & 2.890 \\
\hline Damgard's multi-bit & 25.80 & 128.40 & 241.70 & 0.052 & 0.098 & 0.128 \\
& & & & & & \\
\hline Our multi-bit scheme & 15.60 & 45.30 & 102.10 & 0.11 & 0.221 & 0.258 \\
\hline
\end{tabular}

Big data possess numerous security risks in data collection, storage, and use, which carry with it significant privacy issues with private user data. The accomplishment of security and privacy in the big data world is difficult. A single-bit public key encryption scheme focused on a version of learning parity with noise (LPN) and generalized it to a multi-bit public key encryption scheme in order to meet the growing demand for public key encryption in that area. The correctness of the proposed method is selected plaintext attack security, these schemes have solved encoding error rate problems of the existing LPN-based public key schemes, and the rate of encoding errors in our schemes is negligible [14].

Failure correction is one of the basic problems of machine science theory and has been a central feature in post-quantum cryptography in recent years. The quantum sample complexity of Errors learning and we demonstrate that there is an effective quantum learning method for learning with errors when the error distribution is used in cryptography (with polynomial sample and time complexity).

Quantum learning algorithms do not crack LWE encryption schemes provided by the literature of cryptography; they have some important implications for cryptography: first, one has to pay attention to access to the public key generation algorithm given to the opponent while constructing a LWE-based system; second, our algorithm reveals the possible way to assault a LWE-based e-algorithm [15].
QIP theory, weaker but more practical quantum machines are necessary to solve fascinating but classically difficult problems. Another of the issues with LPN is the noisy quantum unit. In order to show quantum superiority, it is necessary for the LPN question to exploit and to quantify the consistency consumed by one source portion. This also motivates research in the future how related techniques in short-term quantum systems can be used to do more than conventional computing activities and, if any. By utilizing accuracy with many qubits, how much can be enhanced [13].

A variety of cryptographical implementations, such as authentication protocols, pseudorandom generating / function and asymmetric tasks, including PKE (public-key encryption) and obscure transfer (OT), were identified for the recent LPN problem. Learning Noise Parity (LPN) but if, LPN contains collidence-resistant hash (CRH) functions remains a long-standing open question. 
Tarasvi Lakum et al., International Journal of Emerging Trends in Engineering Research, 8(10), October 2020, 6859 - 6863

which parallelly tests only a single layer of small-domain random algorithm (or random permutations) [16].

An analysis of cloud storage, security problems. This seeks to address the key data security concerns posed by the cloud world. These questions were then classified into three categories: 1-data security concerns occurring in relation to conventional infrastructures with the single cloud containing features, 2-data security issues posed in cloud storage across the application life cycle (data processed, utilized and transferred), 3-data security issues correlated with data security qualities, such as confidentiality, privacy, and usability. Different approaches were stressed for every group in order to protect cloud data.[17]

The probability distribution of odd and even bits are ordered based on the key generation, the process of odd and even bits resolving is the solution of DLPN attacker problems, thus, the proposed scheme provides more correctness and security proof. Through the learning parity with noise (LPN), DLPN and RSA algorithms, the proposed system is evaluated, to measure the encryption time, public key and ciphertext bits.[18]

\section{CONCLUSION}

The schemes which are discussed in this review, helpful for the researchers who are working on LPN to overcome the limitations and achieve their targets in cloud environment.

\section{REFERENCES}

1. Avrim blum, adam kalai, and hal wasserman. Noise-Tolerant Learning, the Parity Problem, and the Statistical Query Model. Journal of the acm. (50) 4, 506-519(2003).

2. Henri Gilbert, Matthew J. B. Robshaw, and Yannick Seurin. How to encrypt with the LPN problem. 35 th International Colloquium, ICALP 2008, Reykjavik,Iceland, July 7-11, 2008, Proceedings, Part II Track B: Logic, Semantics, and Theory of Programming \& Track C: Security and Cryptography Foundations, Springer , Lecture Notes in Computer Science, (5126), 679-690 (2008)

3. Sonia Mihaela Bogos. The Learning Parity with Noise Problem. LASEC, I\&C, EPFL. EDIC RESEARCH PROPOSAL. EDIC-ru/05.05(2009).

4. Sanjeev Arora, Rong Ge. Learning Parities with Structured Noise. Electronic Colloquium on Computational Complexity, Report No. 66 (2010).

5. Elena Grigorescu, Lev Reyzin, and Santosh Vempala. On

Noise-Tolerant Learning of Sparse Parities and Related Problems. International Conference on Algorithmic Learning Theory. Algorithmic Learning Theory. 413- 424(2011).

6. Krzysztof Pietrzak. Cryptography from Learning Parity with Noise. SOFSEM 2012, LNCS (7147) 99- 114(2012).
7. Alexandre Duc and Serge Vaudenay. HELEN: a Public-key Cryptosystem Based on the LPN Problem. International Conference on Cryptology in Africa. Progress in cryptology-Africacrypt. 107-126(2013).

8. Arnab Bhattacharyya, Ameet Gadekar, Suprovat Ghoshal, Rishi Saket. On the Hardness of Learning Sparse Parities. Electronic Colloquium on Computational Complexity, Report No. 193 (2015).

9. I. Damgård and S. Park. Cryptol. Res., Tech. Rep. 'How practical is public-key encryption based on LPN and ring-LPN?" Cryptol. [Online]. Available: http://eprint.iacr.org/2012/699.pdf (2016).

10. Peter Hudoba. Public key cryptography based on the clique and learning parity with noise problems for postquantum Cryptography. WSPS3. 20 (2016).

11. Francesco Berti and Francescois-Xavier Standaert. An Analysis of the Learning Parity with Noise Assumption against Fault Attacks. "15th International Conference on Smart Card Research and Advanced Applications (CARDIS 2016)", Cannes (France) (du 07/11/2016 au 09/11/2016).

12. Hanlin Liu, Di Yan, Yu Yu, and Shuoyao Zhao. On the Hardness of Sparsely Learning Parity with Noise. LNCS 10592, 261-267( 2017).

13. Daniel K. Park, June-Koo K. Rhee, and Soonchil Lee. Noise-tolerant parity learning with one quantum bit. Phys. Rev. A 97, 032327 (2018)

14. Zhimin Yu, Chong-Zhi Gao, Zhengjun Jing,Brij Bhooshan Gupta, Qiuru Cai. A Practical Public Key Encryption Scheme Based on Learning Parity With Noise. IEEE Access (6), 31918-31923(2018).

15. Alex B. Grilo and Iordanis Kerenidisy. Learning with Errors is easy with quantum samples. Phys. Rev. A 99, 032314 (2019).

16. Yu Yu, Jiang Zhang, Jian Weng, Chun Guo, and Xiangxue Li. Collision Resistant Hashing from Learning Parity With Noise. Advances in cryptology-ASIACRYPT (2019).

17. Tarasvi Lakum, B.Thirumala Rao. Data Security in Cloud Computing: A Survey. IJARSET. 7, 9, (2020).

18. Tarasvi Lakum, B.Thirumala Rao. A Key-Ordered Decisional Learning Parity with Noise (DLPN) Scheme for Public Key Encryption Scheme in Cloud Computing. (IJACSA) International Journal of Advanced Computer Science and Applications, Vol. 10, No. 11, (2019). 